\title{
HACIA UN ESTUDIO NO-MODULAR DEL LENGUAJE
}

Tim Read

Elena Bárcena

\section{Introducción}

La perspectiva estándar sobre el funcionamiento de la mente en Inteligencia Artificial, Psícología y Ciencia Cognitiva es modular. El uso del término 'modularidad' está relacionado (implícita o explícitamente, según la teoría) con la Hipótesis de la Modularidad de Fodor (1983), según la cual el cerebro tiene un sistema de procesamiento central responsable del razonamiento (con su propia memoria y procesos asociados) y una serie de módulos de input cognitivos separados. Cada módulo es autosuficiente desde el punto de vista funcional, tomando un determinado tipo de input y produciendo un determinado tipo de output. Los módulos de input incluyen la visión, el lenguaje, la planificación, etc. Los procesos y representaciones internas de un módulo no son, por definición, accesibles fuera de ese modulo. Utilizando un ejemplo de Sloman (1989), podemos decir que la estructura arquitectónica del cerebro es comparable a la de un girasol: los pétalos representan los módulos de input separados y el disco central, el procesador.

La noción de modularidad debe ser precisada mediante la introducción de dos términos relacionados: 'macro-modularidad' y 'micro-modularidad'. La macro-modularidad se refiere a la modularización que caracteriza a los procesos de input de dominio específico (o macro-módulos), como la visión, el lenguaje y la planificación. La micro-modularidad se refiere a la modularización de los sub-procesos (o 'micro-módulos') dentro de los macromódulos, e.g., el procesamiento sintáctico y semántico dentro del lenguaje. Estos dos tipos de módulo obedecen por igual los principios generales de la modularidad.

La visión y el lenguaje en concreto han sido estudiados como módulos funcionales autónomos por un gran número de autores como Marr (1982) y Chomsky (1957) respectivamente. Sin embargo, esta perspectiva ha sido rebatida por otros autores, incluyendo Sloman ([1989], con respecto a la visión) y Massaro ([1987], con respecto al lenguaje), quienes defienden la existencia de un traslapo entre los sistemas subyacentes en facultades como la visión, el lenguaje y la planificación. Además, estudios sobre el funcionamiento del cerebro muestran interesantes diferencias en el procesamiento de 
información de los hemisferios izquierdo y derecho y similaridades de activación de estructuras hemisféricas relacionadas en actividades mentales distintas. Consecuentemente, si la demarcación entre sistemas no está tan clara como se había argüido, entonces quizás, por ejemplo, las teorías lingüísticas no constituyen especificaciones de cómo opera un sistema mental separado, sino que son simplemente una descripción de una parte de la arquitectura mental subyacente.

Este artículo estudia las perspectivas modular y no-modular del cerebro concentrándose en la visión y el lenguaje, ya que existe un gran volumen de investigación sobre estos sistemas $\mathrm{y}$, como argüiremos más adelante, hay considerables similitudes, paralelismos e interacciones entre ambos (tomados como módulos de input) que hacen ver fácilmente la posibilidad de un traslapo funcional. Sobre la base de tal traslapo se formula una propuesta para un estudio combinado de la visión y el lenguaje y la búsqueda de una arquitectura subyacente común.

\section{El enfoque modular de la visión}

Durante mucho tiempo se ha considerado la visión como un proceso modular, en el sentido de que su mecanismo es equiparable al de una unidad autónoma y autosuficiente, que desempeña una función por sí misma (y puede ser unida a otros módulos para formar un sistema mayor). Así pues, los mecanismos de la visión toman információn óptica como input y proporcionan tres estructuras dimensionales (incluyendo el movimiento, color, posición, etc.) para ser utilizadas por otros subsistemas.

Se puede sostener que el defensor más conocido de esta perspectiva ha sido Marr. En su famoso libro (1982), desarrolló una teoría de la visión que ha motivado desde entonces un gran volumen de investigación sobre sistemas de visión artificial. Este sistema funciona básicamente del siguiente modo: la información llega del mundo en forma de rayos de luz que forman patrones (de variada intensidad y color) en la retina ocular. El procesamiento inicial de esta información por parte del sistema nervioso produce el esquema primitivo bruto, que consiste principalmente en mapas de cambios de intensidad y color. Estos mapas son entonces agrupados por medio de procesos recursivos en estructuras de mayor escala, las cuales forman el esquema primitivo completo. El esquema de dimensión 2.5 incluye información profunda y el modelo tridimensional consiste en objetos localizados en el espacio, cuyas estructuras están disponibles para ser accedidas por otros subsistemas para tareas como su identificación verbal.

Marr proporciona el ejemplo de un gato, para el que el esquema primitivo bruto consistiría en mapas de pelos individuales, el esquema primitivo completo, el patrón del pelaje, etc., hasta que un objeto completo que representa al gato es almacenado como una representación tridimensional en el nivel más alto de la jerarquía. El principal rasgo de la teoría de Marr es que los objetos están representados de modo jerárquico y combinatorio.

Una perspectiva de la visión tan modular ha sido confontrada por varios autores como (indirectamente) Gibson (1986) y más tarde Sloman (1989). Gibson considera la visión en términos de las propiedades que ofrece la información visual, y no según las 
consideraciones geométricas de Marr. Sloman añade que los requisitos de la visión humana se satisfacen mejor por un sistema heterárquico distribuido funcionalmente e interconectado fuertemente con otros procesos mentales de modo que no se puede hacer una clara demarcación entre sistemas.

\section{El enfoque modular del lenguaje}

El panorama lingüístico actual está formado por un considerable número de teorías gramaticales heterogéneas con perspectivas formalistas o funcionalistas y metodologías descriptivas bien distintas. Ejemplos de tales teorías son la Teoría de la Rección y el Ligamento (GB) (Chomsky 1981), la Gramática Léxico-Funcional (LFG) (Kaplan \& Bresnan 1982), la Gramática de Estructura Sintagmática de Cabecera (HPSG) (Pollard 1984) y la Gramática Funcional (Dik 1979). Sin embargo, todas ellas son macro-modulares en el sentido de que explican el proceso comunicativo verbal a través de elementos y funciones propiamente lingüísticos, sin recurrir a otros sistemas cognitivos.

Se puede hablar de que hay una tradición de investigación que ve los niveles lingüísticos (fonología, morfosintaxis, semántica y pragmática) como autónomos e independientes. Así pues, se llega a la representación completa de una ocurrencia lingüistica dada a partir de la aplicación gradual de distintos tipos de conocimiento que no intervienen entre sí. Esto quiere decir que la información semántica y contextual no influye durante el funcionamiento del procesador sintáctico (Frazier 1978) y, por tanto, la ambigüedad estructural es, en la medida de lo posible, resuelta sin recurrir a információn no-sintáctica (Stevenson 1993). Este tipo de modelo de análisis sintáctico 'modular' o 'independiente' no es el único, pero ha de tenerse en cuenta que hay modelos considerados 'interactivos' que actuan sobre el output de otro módulo, e.g., la semántica actua sobre el output del procesador sintáctico para eliminar falsos análisis en base a la nueva información. Por tanto se mantiene la modularidad.

\section{El traslapo entre la visión y el lenguaje}

Los mecanismos de la visión y el lenguaje conllevan una serie de representaciones y procesos que incluyen los siguientes:

- visión: contorno, textura, color, mapas de intensidad; flujo óptico; bases de datos de bordes, líneas, regiones, disparidades binoculares; información sobre la forma; agrupaciones en estructuras mayores; interpretaciones en términos de forma y movimiento tridimensionales; construcción de relaciones: espacial, causal, funcional e intencional.

- lenguaje: fonemas, letras; morfemas, palabras; claúsulas, oraciones; discurso, texto; análisis sintácticos; interpretaciones semánticas a nivel léxico y estructural; estructura retórica, dependencias contextuales, comprensión, construcción de relaciones e inferencias.

Estas representaciones y procesos pueden verse como una primera indicación de la nomodularidad de la visión y el lenguaje, así como de su intersección. Primero, ambos sistemas están formados por partes bien diferenciadas. Segundo, son jerárquicos, i.e., en ambos casos dichas partes se forman a partir de estructuras más simples. Tercero, los 
procesos de combinación de las representaciones son generativos, i.e., dado un conjunto finito de textura, color y patrones de intensidad (en el caso de la visión) y de fonemas y letras (en el caso de la lengua), se puede generar una variedad de estructuras de orden superior practicamente infinita. El proceso de combinación de las representaciones es similar en cada caso y cabe pensar que podría explicarse por medio de un mecanismo de ensamblaje genérico, utilizando diferentes conjuntos de reglas para las dos operaciones (como ha sido ya sugerido por Corballis [1991]). Cuarto, se puede observar que algunos procesos que intervienen en la visión y el lenguaje se encuentran fuera del dominio visual y lingüístico propiamente dichos, así como un área de procesamiento común entre ambos sistemas.

\subsection{Evidencia psicológica experimental}

El test de Stroop es uno de los experimentos más clásicos de psicología. Se les presenta a los sujetos una serie de palabras que denotan colores, cada una de las cuales aparece escrita en un color, que puede o no corresponder al color designado por la palabra. Entonces se les pide que nombren el color en el que está escrita cada palabra y se calcula el tiempo tardan en hacerlo, e.g.:

- La palabra ROJO está escrita en aparece rojo (el sujeto dice rojo)

- La palabra AZUL está escrita en amarillo (el sujeto dice amarillo)

- La palabra ROJO está escrita en azul (el sujeto dice azul)

- La palabra VERDE está escrita en rojo (el sujeto dice rojo)

El efecto Stroop se refiere al hecho de que un sujeto tarda más en nombrar el color en el que está escrita una palabra coloreada cuando los colores no coinciden. Este efecto ilustra algún tipo de interferencia entre el proceso visual de la percepción cromática y el proceso lingüístico de la lectura. Si los sub-sistemas responsables de la visión y la lengua no estuvieran interconectados, no habría lugar para tal interferencia. Esto sugiere un traslapo estructural y funcional en los mecanismos subyacentes correspondientes.

En situaciones en las que una imagen es ambigua, la información lingüística proporcionada al sujeto puede ayudar a desambiguar, casi subconscientemente, lo que se está viendo. Las siguientes imágenes intentan ilustrar la desambiguación visual:

Tras alguna deliberación, que seguramente no ayudará al lector a identificar las imágenes, la información lingüística de que la imagen de la izquierda es un mejicano montando en bicicleta y la de la derecha una jirafa pasando por delante de una ventana probablemente ayudará al lector a identificar las imágenes confusas. Este ejemplo vuelve a indicar la existencia de un traslapo estructural y funcional en los mecanismos subyacentes entre la visión y el lenguaje, de modo que la información de un tipo puede asistir al procesamiento del otro tipo de información. 


\subsection{Evidencia neuropsicológica}

Existen técnicas muy variadas para permitir el estudio directo del cerebro, en su funcionamiento normal y anormal. Hay, por ejemplo, técnicas quirúrgicas básicas que sirven para estudiar post mortem la degeneración de las estructuras del cerebro de individuos que han tenido problemas mentales (tal técnica ha sido utilizada por investigadores como Broca [1861]). También se han desarrollado técnicas de estudio de cerebros divididos por investigadores como Gazinger (1970) y Sperry (1974), con el fin de examinar el distinto rol de los hemisferios derecho e izquierdo en las funciones mentales. Además, el trabajo con pacientes con enfermedades degenerativas como el alzheimer permiten el estudio del rol de las distintas estructuras del cerebro (Appel et al. 1982; Foster et al. 1986). También son muy útiles las técnicas de imágenes del cerebro no-intrusas como PET (Tomografía de Emisión de Positrón) y MRI (Figuración de Resonancia Mágnética), que proporcionan información sobre la localización y activación de estructuras mentales en distintos procesos. Dadas tales técnicas y años de estudio, los síndromes siguientes han podido ser identificados, junto con sus localizaciones en el cerebro. Los desórdenes visuales están relacionados con tipos de 'agnosia', que es el nombre empleado en neuropsicología para denominar al fenómeno general que incapacita el reconocimiento de reconocer objetos familiares. Los desórdenes lingüísticos están asociados con la 'afasia', que es el término específico para denominar el desorden general del lenguaje. El siguiente resumen está basado en el trabajo de Corballis (1991):

- Agnosia asociativa: un deterioro en el que los pacientes son capaces de separar unos objetos de otros y de percibirlos en tres dimensiones, pero no de nombrarlos o de asociarles un significado. Hay dos formas extremas de agnosia asociativa: la alexia o incapacidad para leer palabras impresas y la prosopagnosia o incapacidad para reconocer caras conocidas. La primera condición se relaciona generalmente con daños en el hemisferio izquierdo y la segunda, con daños en el hemisferio derecho.

- Agnosia perceptiva: una dificultad en reconocer objetos cuando se presentan de modos complejos, como en forma de dibujos lineares superpuestos o siluetas. Está relacionada con daños en la parte posterior del hemisferio derecho, más que en el izquierdo.

- Pseudoagnosia: un rompimiento o interrupción general de las primeras fases de la visión, más que un problema relativo al procesamiento de objetos. Está asociada principalmente con daños en el hemisferio derecho.

- Afasia de Broca (o de motor): un deterioro en la producción del lenguaje, pero no en la comprensión. Está relacionada con daños en el área de Broca, una parte de la zona posterior del lóbulo frontal, cercano a la corteza motora.

- Afasia sensorial (o de Wernicke): un deterioro en la comprensión del lenguaje, pero no la producción (incluso la comprensión del propio habla del paciente es imposible). Está asociada con daños en el área de Wernicke, una parte de la zona posterior del lóbulo temporal, alrededor de la juntura de los lóbulos temporal, parietal y occipital.

- Afasia de conducción: un error en la sustitución de fonemas en las palabras. Un 
ejemplo que proporciona Corballis (1991) es: fots of fun en lugar de lots of fun. Además, el paciente es incapaz de repetir lo que acaba de escuchar. Está relacionada con daños en las fibras que conectan el área de Broca con el área de Wernicke.

- Afasia transcortal: una categoría de problemas que tienen en común que el paciente repite automáticamente todo lo que escucha. Estas condiciones están relacionadas con daños cerca del área de Broca o de Wernicke.

Tras esta breve revisión de patologías neuropsicológicas se puede deducir que en general los deterioros lingüísticos están causados por daños a estructuras en el hemisferio izquierdo y los deterioros visuales, por daños en ambos hemisferios. Sin embargo, parafraseando la propia conclusión de Corballis (1991) sobre los desórdenes visuales, se puede establecer una distinción entre las condiciones de agnosia generativa basada en partes (como la alexia), que son causadas por daños en el hemisferio izquierdo, y las condiciones de agnosia holística (como la prosopagnosia), que surgen de daños en el hemisferio derecho. Corballis presenta el argumento de que hay un recurso de ensamblaje generativo basado en el hemisferio izquierdo que es responsable del ensamblaje basado en partes de un vocabulario fijo de elementos (tal y como está presente en el lenguaje y en la visión basada en objeto) y un sistema de representación holística de apoyo basado en el hemisferio derecho (importante en el procesamiento de la lengua escrita y de las estructuras visuales complejas). Sloman (1989) añade en este sentido que la visión cońlleva un 'análisis sintáctico', en cuanto que las estructuras jerárquicas son halladas en las imágenes del mismo modo que un analizador sintáctico encuentra estructuras en las oraciones (Fu 1977).

\section{El enfoque laberíntico dell lenguaje}

Los enfoques basados en el diseño (Sloman 1993) tienen como objetivo la producción de teorías adoptando lo que se conoce como la 'plataforma de diseño' (design-stance) (Dennet 1978), aspirando a explicar los principios de un sistema desde la perspectiva de su diseño. Se trata de considerar qué requisitos ha de satisfacer el sistema, las restricciones presentes en sus posibles diseños y el modo en que los mecanismos que forman el sistema ejecutan el conjunto de los requisitos y las restricciones. Un ejemplo de este enfoque puede verse en la cuestión de cómo el cerebro trata el reconocimiento de palabras ante un input ambiguo. Se sabe que la identificación de una letra en una palabra puede influir en la identificación de otra si esta última no puede ser directamente reconocida en el input inicial. McClelland \& Rumelhart (1981) añadieron a esta cuestión la restricción de que el diseño de un sistema capaz de explicar tales comportamientos debería estar basado en la tecnología de redes neuronales, ya que asumían que el funcionamiento del cerebro está determinado por su estructura neuronal subyacente. Dados tales requisitos y restricciones (se consideraron otros que no están incluidos aquí), estos autores fueron capaces de producir un diseño y su correspondiente modelo computacional, el cual ejecutó con éxito los datos experimentales disponibles sobre este fenómeno.

Dadas las nociones de diseño en general y de enfoque basado en el diseño en particular, se puede hacer una distinción entre 'diseños estrechos y profundos' y 'diseños anchos y superficiales’ (Bates et al. 1991). Los primeros constituyen el tipo estándar de diseños 
producidos por investigadores que intentan explicar en gran detalle el funcionamiento de unos pocos mecanismos relacionados, mientras que los segundos intentan explicar el funcionamiento de muchos mecanismos relacionados en menor detalle, aunque el objetivo último de este tipo de investigación es llegar a desarrollar diseños anchos y profundos. Por supuesto, hay lugar para ambos tipos de enfoque en la comunidad investigadora. El enfoque basado en el diseño (conducente a cualquiera de los dos tipos) es una herramienta poderosa que permite que se exploren las capacidades mecanísticas, se especifiquen claras relaciones entre los requisitos, restricciones y diseños y, si se requiere, se prueben matemáticamente.

La noción de una teoría laberíntica fue introducida por primera vez por Sloman (1989). $\mathrm{Su}$ argumentación partía de la identificación de los errores de las perspectivas previas modulares de la visión hacia un enfoque más global. El defendía que las facultades como la visión, la planificación, etc. son facetas de una arquitectura común (y no módulos separados que pueden entenderse exlusivamente en términos de su propio funcionamiento interno). El enfoque laberíntico de la visión estudia qué tipo de diseño (y de simulación computacional) habría que construir para explicar la capacidad visual sobre la base de la existencia de una sola arquitectura cerebral subyacente, común a todas las facultades mentales. La visión es parte de una arquitectura heterárquica integrada de mayor escala, donde no se pueden dibujar límites de módulos simples. Su diseño necesita ser ancho y superficial para permitir que se estudien simultaneamente la gran gama de requisitos de los distintos procesos cognitivos y que este objetivo investigador sea viable y realista.

En cuanto al lenguaje, cabe hacer dos observaciones. Primero, los argumentos nomodulares de Sloman sobre la visión son aplicables al lenguaje. Segundo, el estudio de los requisitos mutuos de ambos sistemas proporciona claves sobre la naturaleza de los mecanismos y las representaciones comunes subyacentes. Debido a la interacción entre la visión y el lenguaje, se puede esperar que la dirección de investigación del diseño de la visión sugerida por Sloman lleve efectivamente a una explicación del lenguaje y que en dicho diseño ambas facultades tengan numerosos puntos de interconexión.

Así pues, uniendo la propuesta de un enfoque basado en el diseño (en el contexto de diseños 'anchos y superficiales') y la evidencia existente de la intersección entre la visión y el lenguaje, proponemos adoptar un enfoque laberíntico del lenguaje. Este enfoque tiene como primer objetivo explicar el lenguaje, pero también se aspira a hacer descubrimientos significativos sobre la natualeza de la representación y el procesamiento de la información. Se pretende estudiar la integración funcional de la información visual y lingüística en representaciones comunes sofisticadas y adaptivas, cuyos resultados puedan proporcionar descubrimientos sobre el modo en el que el nuevo input sensorial del mundo se integra con las representaciones existentes, para tratar los requisitos visuales y lingüísticos (e.g., la desambiguación visual a través de input lingüístico, diciendo lo que el cuadro representa realmente, y la desambiguación lingüística a través de input visual, como la inclusión de diagramas entre los párrafos de un texto). Además, tal proceso de diseño podría explorar la Hipótesis de Corballis (1991) sobre la existencia de un mecanismo de ensamblaje generativo basado en el hemisferio izquierdo del cerebro y su sistema de apoyo de representación holística basado en el hemisferio derecho. 


\section{Conclusión}

Este artículo empezó observando que la investigación actual sobre el funcionamiento de la mente apoya a menudo la Hipótesis de la Modularidad, bien sea explícita o implícitamente. Se precisó que el concepto de modularidad existe de dos formas; así pues, distinguimos entre la macro-modularidad (según la cual se asume que los sistemas de input como la visión y el lenguaje son módulos funcionales independientes) y la micromodularidad (según la cual los sub-procesos de un módulo pueden considerarse módulos a su vez dentro de un sistema mayor, como los módulos de procesamiento sintáctico y semántico dentro del módulo del lenguaje). Las teorías de la visión y el lenguaje como las de Marr y Chomsky respectivamente se caracterizan por su modularidad en ambos sentidos, ya que intentan explicar los funcionamientos sistémicos correspondientes en términos de sus propias representaciones y procesos internos, los cuales funcionan independientemente del resto de los sistemas cognitivos.

A continuación se presentaron argumentos que defendían que la visión y el lenguaje no son modulares, sino que tienen mecanismos comunes que forman parte de una arquitectura subyacente integrada. Estos argumentos se basaban en análisis teóricos de la estructura de las representaciones y los procesos visuales y lingüísticos y en fenómenos observados empíricamente en experimentos psicológicos comunes o en casos de patología neuropsicológica. Dichos mecanismos han sido interpretados como un claro ejemplo de la interacción existente entre las facultades humanas. Este artículo ha presentado el enfoque laberíntico como un método realista y eficiente de llegar a una explicación del funcionamiento del lenguaje a través del desarrollo de diseños más globales e interactivos de representaciones y procesos cognitivos.

\section{Referencias}

- J. Appel, A. Kertesz \& M. Fishman, "A study of language functioning in Alzheimer patients”, Brain and Language 17 (1982) 73-91.

- J. Bates, A.B. Loyall \& W.S. Reilly, "Broad Agents", SIGART Bulletin (1991).

- P. Broca, Remarques sur la siege de la faculté du langage articulé, suiviés d'une observation d'aphemie, Bulletin de la Societé anatomique de Paris 2 (1861) 330357.

- N. Chomsky, Syntactic Structures (The Hague 1957).

- N. Chomsky, "Lectures on Government and Binding Theory: The Pisa Lectures", Studies in Generative Grammar 9 (1981).

- M.C. Corballis, The Lopsided Ape, Evolution of the Generative Mind (Oxford 1991).

- D.C. Dennet, Brainstorms (Bradford 1978).

- S.C. Dik, Functional Grammar (Amsterdam 1978).

- J. Fodor, Modularity of Mind (Cambridge 1983). 
L. Frazier, On comprehending sentences: syntactic parsing strategies (Connecticut 1978).

- N.L. Foster, T.N. Chase, N.J. Patronas, R.N. Gillespie \& P. Fedio, "Cerebral mapping of apraxia in Alzheimer's Disease by positron emission tomography", Annals of Neurology 19 (1986) 139-143.

- K.S. Fu, Syntactic Pattern Recognition Applications (Berlin 1977).

- M.S. Gazzaniga, The Bisected Brain (New York 1970).

- J.J. Gibson, The Ecological Approach to Visual Perception (1986).

- R. Kaplan \& J. Bresnan, "Lexical-Functional Grammar: A Formal System for Grammatical Representation", en J. Bresnan (ed.) The Mental Representation of Grammatical Relations (Cambridge 1982).

- D. Marr, Vision (San Fransisco 1982).

- D.W. Massaro, Speech perception by ear and eye: A paradigm for psychological inquiry (New Jersey 1987).

- J.L McClelland \& D.E. Rummelhard, "An interactive activation model of context effects in letter perception: part 1. An account of basic findings", Psychological Review 88 (1981) 375-407.

- C. Pollard, Generalised Phrase Structure Grammars, Head Grammars, and Natural Language (Standford 1984).

- R.W. Sperry "Lateral specialization in the surgically separated hemispheres", en F.O. Schmitt y F.G. Worden (eds.) The Neurosciences: Third Study Program (1974).

- A. Sloman, "On designing a vision system (Towards a Gibsonian computational model of vision)", Journal of Experimental and Theoretical Artificial Intelligence 1 (Cambridge 1989) 289-337.

- A. Sloman, "Prospects for AI as the general science of intelligence", en Proceedings of AISB 93 (Amsterdam 1993).

- R.J. Stevenson, Language, Thought, and Representation (1993). 



\section{NORMAS DE PUBLICACIÓN}

1. Los originales deberán ser inéditos y no estar aprobados para su publicación por ninguna otra entidad.

2. Los originales recibidos serán examinados por miembros del Consejo de Redacción y/o del Consejo Asesor de la Revista.

3. La publicación de artículos en las revistas de la Universidad de Sevilla no da derecho a remuneración alguna. Los derechos de edición son de la Facultad de Filología de la Universidad de Sevilla y es necesario su permiso para cualquier reproducción. En todo caso, será preciso citar la procedencia en cualquier reproducción parcial o total.

5. Los autores tendrán derecho a veinte separatas de los artículos y tres de las reseñas.

\section{NORMAS DE PRESENTACIÓN}

1. Los originales se enviarán al Secretario de la revista (Facultad de Filología, Departamento de Lengua Española, Lingüística y Teoría de la Literatura, C/ Palos de la Frontera, s/n, 41004 Sevilla). Irán precedidos de una hoja en la que figure el título del trabajo, el nombre del autor (o autores), su dirección y el teléfono y el nombre de la institución a la que pertenece. Asimismo, se hará constar la fecha de envío del trabajo.

2. Los artículos y notas tendrán una extensión máxima de veinticinco y ocho páginas respectivamente, a razón de treinta líneas por página y setenta caracteres por línea como máximo. Las reseñas tendrán una extensión máxima de tres páginas. El autor especificará si presenta el trabajo en calidad de artículo o de nota. En todo caso, el Consejo de Redacción se reserva el derecho de proponer al autor la inclusión de su trabajo en una sección distinta de la indicada.

3. Se ha de enviar dos copias impresas de cada manuscrito. Debe adjuntarse una copia del documento original en soporte informático, indicando en la portada del disco el Sistema Operativo y el nombre y versión de la aplicación que creó el documento. Se aceptarán disquettes preferiblemente de 3'5 pulgadas formateados en sistema Mac o MS-DOS. Pueden enviarse ficheros creados con las aplicaciones siguientes: WORDPERFECT, MS WORD, LOTUS AMIPRO o WORDPRO'97, todas ellas en versión McIntosh o Windows, pero sólo se aceptarán disquetes de 3'5 pulgadas formateados en sistema MS-DOS para PC. Los ficheros de texto ASCII también son aceptados, debiendo llevar las notas a pie de página enumeradas consecutivamente al final del trabajo.

4. Cada artículo irá acompañado de un resumen de no más de quince líneas (aprox. 250 palabras) redactado en inglés, en hoja aparte (dos copias).

5. Los cuadros, mapas, gráficos, tablas, figuras, etc., que se entreguen con los trabajos deberán ser originales y se presentarán preferentemente en papel vegetal y perfectamente rotulados. Todos irán numerados y llevarán un breve pie o leyenda para su identificación. También se indicará el lugar aproximado de su colocación.

\section{NORMALIZACIÓN DE CITAS Y NOTAS}

1. Los números indicativos de nota serán numerales arábigos, escritos ligeramente por encima de la línea de escritura, e inmediatamente después del material al que se refiere. No irán entre paréntesis.

2. Si la referencia es breve, puede insertarse entre paréntesis en el texto (Ej. Eliot 1920:37), pero la primera referencia completa de un trabajo debe aparecer en una nota o al final del trabajo.

3. Las citas bibliográficas atenderán a las normas siguientes:

LIBROS: INICIAL. APELLIDO DEL AUTOR, TÍTULO (CIUDAD AÑO) PÁGINAS

Ejemplo: T. S. Eliot, The Sacred Wood (London 1920) 36-38.

REVISTAS: INICIAL. APELLIDO DEL AUTOR, "TÍTULO DEL ARTÍCULO", REVISTA No (AÑO) PÁGINAS

Ejemplo: A. Díaz Tejera, "El género en griego clásico: descripción sincrónica 'y explicación diacrónica", Revista Española de Lingüística 11 (1981) 13-31. 


